\title{
Fibre and water binding
}

\author{
Martin F. Chaplin \\ School of Applied Science, South Bank University, Borough Road, London SE1 OAA, UK
}

\begin{abstract}
The range of interactions between fibre and water and the consequential properties of the bound water are modelled and examined. Dietary fibre may interact with water by means of polar and hydrophobic interactions, hydrogen bonding and enclosure. The results of these interactions vary with the flexibility of the fibre surface. When the fibre is insoluble or junction zones are formed, this may result in profound changes in the surrounding water. Such interactions are capable of affecting the structuring and solvation properties of water well away from the immediate surfaces involved. In particular, the specific properties of water enclosed by dietary fibre are examined, an area of investigation previously receiving scant attention. The way this enclosure may affect the properties of water is exemplified by modelling the colon to show how fibre may exert a beneficial action by the preferential partitioning of hydrophobic carcinogens. Unfermented dietary fibre has a tendency to form low-density expanded water that acts as a preferential solvent for hydrophobic molecules when compared with the less-structured denser water within the much more hydrophilic mucus layer.
\end{abstract}

Dietary fibre: Water: Hydration: Colon: Aqueous biphasic partition

\section{Importance of fibre and water}

Dietary fibre is generally accepted as having protective effects against a range of diseases predominant in Western developed countries (Hill, 1999). The major components are polysaccharides that reach the colon undigested. These polysaccharides consist of the NSP, including cellulose, hemicellulose (composed of a variety of heteropolysaccharides including arabinoxylans), $\beta$-glucan, pectins and added hydrocolloids, plus undigested starch. Whilst the physiological properties of these polysaccharides are difficult to predict on the basis of their structures alone, they are partly predictable on the basis of physico-chemical properties such as fermentation, water-holding capacity, viscosity and bile acid binding. They are dependent on the site, rate and extent to which they are absorbed or fermented in the digestive tract. Fermentation, which is greater when there is a greater surface:volume, lowers the colonic $\mathrm{pH}$ and allows changes in the gut flora to one less prone to produce carcinogens. It additionally causes changes to the properties of the fibre and may remove it altogether (Guillon et al. 1995). Consumption of dietary fibre has been found to increase stool weight, alter gut transit time, alter activity of the colonic microflora, influence appetite, absorb toxins and modify the absorption of fats, sugars, minerals and bile acids. The extent to which specific polysaccharides and hydrocolloids exert their physiological effects is dependent on a complex mixture of structural, chemical and physical properties (Blackwood et al. 2000).

\section{Type of water binding}

Water binding has a particularly important relevance to the physiological actions of dietary fibre. It is well known that water is bound to polysaccharides with differing strengths and in different amounts. Any polysaccharide may show different strengths of binding with different parts of its structure. Binding may be determined by filtration (waterholding capacity), centrifugation (water-binding capacity) or freeze-drying. Bound water is often divided into two types (freezing and non-freezing) because of the relative ease with which such a determination can be made, by means of enthalpy changes on freezing and melting. The assumption is usually made that non-freezing water is more tightly and specifically bound. It should be noted, however, that all methods for measuring 'bound' water only determine the water according to the method used and should not be generalised to include other binding classes. As examples, water retained by centrifugation depends on the $\boldsymbol{g}$-force used and, therefore, may be a better indicator of centrifugal speed rather than binding strength; also 'non-freezing' water 
indicates only that the water does not freeze. It indicates nothing about the intrinsic binding, as it is generally considered that even ice has a layer of unfrozen water at its surface (Devlin, 2000), and such water may simply be present as a glass without any specific 'binding'.

There are two 'flavours' for unbound water, trapped and free. Generally, as particle size increases so does the trapped volume, due to imperfect packing, and consequential apparent water binding (Thebaudin et al. 1997). Trapped water has decreased freedom of movement (entropy). In order to keep the water activity constant, therefore, the water has to form bonds with a more negative enthalpy. This process results in stronger more-linear hydrogen bonds, causing greater structuring and lower density. The same effect is caused by capillary action; the reduced pressure 'stretching' the water into a more open tetrahedral structure (Bellissent-Funel, 2001).

\section{Interaction of 'dietary fibre' with water}

Fibre can directly bind water in a number of ways (Fig. 1). Enclosure is by far the most important if judged in terms of the volume affected (Auffret et al. 1994). 'Stretched' water is formed where capillary action increases in the presence of strong surface hydrogen bonding. Gas in the colon may also increase this capillarity. Interaction of water with soluble fibres at low concentrations results in the fibre molecules forming extended elongated conformations, where each molecule influences the large volume of water within its effective radius of gyration. If the concentration increases, molecules fold on themselves to avoid the loss of their rotational entropy. At high concentrations, 'compatible' molecules mix but 'incompatible' molecules separate into a biphasic aqueous system. Such phase separation is mainly attributed to 'incompatibility' between the hydration shells of one molecule and that of the other. The different aqueous phases produced may possess considerably different solvation and physico-chemical properties.

\section{Levels of polysaccharide hydration}

Polysaccharide hydrogen bonding may take place (a) intramolecularly between the polysaccharide hydroxyl groups, (b) through a bridging water molecule that is doubly hydrogen bonded to a single polysaccharide chain or (c) entirely through single hydrogen bonding to water molecules that are simultaneously held by up to three hydrogen bonds to other water molecules. Water binding has both enthalpic and entropic effects, and also involves consequential enthalpic and entropic effects between neighbouring water molecules. Where only intramolecular hydrogen bonding occurs, the structure is left with a relatively hydrophobic character giving, for reasons explained later (p. 226), a more expanded aqueous environment. Such intramolecular hydrogen bonds (type a, described above) may often hydrate using a bridging water molecule to form structure type b. However, such doubly-bound water molecules have lost much of their freedom of movement, with the maximum
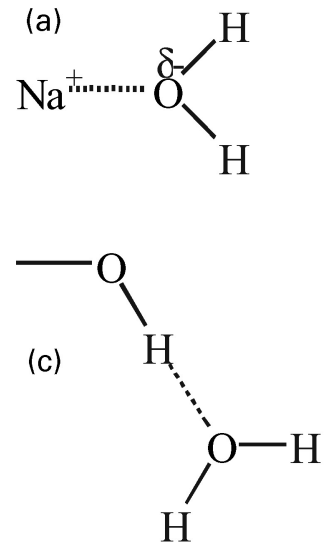

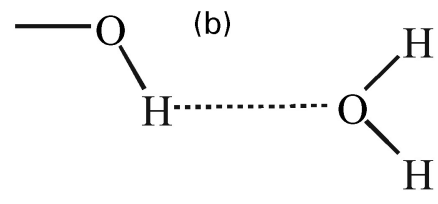

(f)

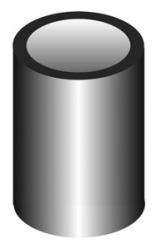<smiles>CC1OC(O)OC2OC(C)OC(O1)O2</smiles>

Fig. 1. Fibre can directly bind water in a number of ways: (a) polar effects involving both anions and cations orient and attract water; (b) weak hydrogen bonding involves bent or extended hydrogen bonds; (c) strong hydrogen bonding involves straight, directed and short hydrogen bonds; (d) ionic interactions involve strongly held and oriented water, here shown through interactions between a carboxylate group and potassium ion; (e) hydrophobic (surface) effects involve water clathrate formation at surfaces where water molecules form a flattish network of pentagons and hexagons; (f) enclosure of water involving capillary action (not to scale). 
entropic energy loss being equivalent to about the enthalpy involved in one hydrogen bond, whilst still behaving in a somewhat hydrophobic manner. Maximum hydration (type c, described earlier) with the greatest freedom of movement, for both the fibre and water molecules, occurs when water molecules only share one hydrogen bond each with the polysaccharide. Such polysaccharide structures, thus, exhibit the greatest hydrophilicity. The presence of polysaccharidic carboxylate groups tend to increase the water density as the distance between the two hydrogen-bonding oxygen atoms $(2 \cdot 2 \AA)$ is markedly less than the $\mathrm{O}-\mathrm{O}$ distance in water $(2 \cdot 8 \AA)$. Cationic counterions may further increase the localised density due to the water clustering close together around their small positive charges. Furanose sugars, as found in arabinoxylans, also tend to create a volume of denser water, as they break up the structure of water surrounding them by constantly making and breaking hydrogen bonds as they flip between their North and South conformations. Highly-flexible glycosidic linkages, such as the $\alpha-1-6$ branch-points in $\alpha$-glucans may exert similar effects.

Aqueous structuring additionally depends on how well the hydrogen bonding can fit into a tetrahedral network of water. As a measure of this fit, the distances between the $1-2,1-3,1-4,2-3,2-4$, and 3-4 O-.O atoms of homopolysaccharides may be compared with the relevant $\mathrm{O} \cdot . \mathrm{O}$ distances found in ice or supercooled water $(2.82 \AA, 4.60 \AA$, $5.40 \AA, 2.82 \AA, 4.60 \AA$ and $2.82 \AA$ respectively). The mean square deviation distances are: $\beta$-D-glucan, $0.02 ; \alpha-\mathrm{D}$ glucan, 0.16 ; $\beta$-D-mannan, 0.03; $\alpha$-D-mannan, 0.29; $\beta$-Dgalactan, $0.18 ; \alpha$-D-galactan, $0.17 ; \beta$-D-xylan, $0.02 ; \alpha-D-$ xylan, $0 \cdot 17$. The molecules that fit best into the expanded water structure ( $\beta$-D-glucan, $\beta$-D-mannan and $\beta$-D-xylan) cause the greatest reduction in the freedom of movement of the water molecules. This process costs energy and, consequentially, the surface minimisation that occurs reduces the solubility of 1-4 $\beta$-glucans, $\beta$-xylans and $\beta$-mannans.

\section{Modelling of gels and the hydration layer}

It is now well established that proteins possess a hydration layer about one molecule deep (Durchschlag \& Zipper, 2001). This layer gives rise to about $0.35 \mathrm{~g} / \mathrm{g}$ bound water that exhibits relatively long residence times, tumbling with the protein. It is of interest to investigate whether a similar hydration layer can explain the hydration properties of the polysaccharides that make up dietary fibre. Although such binding may occur with single chains, it is most likely that the polysaccharide chains should be relatively inflexible if the water is not to be easily shed. A major factor in this binding of water therefore concerns the interaction of the polysaccharide chains to form junction zones. These structures are necessarily stiffer, and not only bind some water strongly but also cause the enclosure of large amounts of water. They have a major effect on the structure and properties of this water, which is consequentially considerably different from the bulk water environment.

In order to examine the effects of the gelling on water, gels were modelled on a cubic mesh of molecules joined by junction zones. These junction zones necessarily restrict the exit of water from the cavities. Various gels were modelled and at $1 \%(\mathrm{w} / \mathrm{w})$ they formed cubic lattices with a radius of about $10 \mathrm{~nm}$. The volume and surface areas of these junction zones were determined by the method of Bodor et al. (1989). Junction zones were found to be denser and less flexible than the single chains (Table 1). Although their formation released considerable surface water into the bulk, the remaining water is fairly static, as it cannot readily move away. This situation effectively increases the fibre diameter of the junction zones, so reducing the pore size, increasing the capillarity and reducing the tendency for the water to exit the cavities. The confined water is much more highly structured than the bulk water (Bellissent-Funel, 2001). In terms of capillary action, if the gels are considered totally inflexible, such pore sizes are able to produce a negative pressure of $\leq 15 \mathrm{MPa}$, and a water activity of about 0.9 . Formation of smaller pores would consequentially produce a substantial amount of non-freezing water simply due to this reduction in water activity. It is generally found that much water is absorbed at the junction zones, which is mostly freezing bound water, and this absorption of water increases with the junction zone formation. If there is more order in the fibre, then there is more order in the water it contains (Hayashi et al. 2002). The surface water binding of cellulose is much lower than the bound water found experimentally for the amorphous solid (Ang, 1991), so indicating the greater amount of water held by enclosure.

Table 1. Model results for the formation of porous cubic gels formed from various polysaccharides* $(1 \%, w / w)$ linked by junction zones

\begin{tabular}{|c|c|c|c|c|}
\hline & \multirow[b]{2}{*}{ Cube side $(\mathrm{nm})$} & \multirow[b]{2}{*}{ Surface water $(\mathrm{g} / \mathrm{g}) \dagger$} & \multicolumn{2}{|c|}{ Polymer: } \\
\hline & & & $\%$ density increase on forming gel & $\%$ surface decrease on forming gel \\
\hline Alginate & 20 & 1.15 & 15 & 21 \\
\hline Amylose & 21 & 0.96 & 25 & 47 \\
\hline Carrageenanł & 22 & 1.03 & 21 & 41 \\
\hline Cellulose & 32 & 0.68 & 48 & 66 \\
\hline Galactomannan & 17 & 1.28 & 17 & 33 \\
\hline Gelatin§ & 21 & 1.22 & 37 & 49 \\
\hline Pectin & 21 & $1 \cdot 24$ & 10 & 17 \\
\hline Xylanll & 15 & 1.55 & 17 & 29 \\
\hline
\end{tabular}

*All polysaccharides utilised two strands for junction-zone formation, except for cellulose which utilised seven strands.

†Compare with proteins having $0.35 \mathrm{~g}$ surface water/g (Durchschlag \& Zipper, 2001).

łJanaswamy \& Chandrasekaran (2001).

$\S$ Chen et al. (1991).

IINieduszynski \& Marchessault (1972). 


\section{Low-density water formation}

In order to understand what happens within the porous cavities formed by the unfermented fibre, the forces holding the water together are considered. These forces are the dipole and induced dipole interactions pulling molecules closer and hydrogen bonds holding them together but oriented and further apart. At surfaces, if the hydrogen bonds are lost then water gets closer to the surface, causing increased density. However, the next layer of water away from the surface will get fuller, stronger and more extensive hydrogen bonding (Wiggins, 1995). In water the tension between these forces results in equilibria between denser and less-dense aqueous clusterings (Chaplin, 2001). More extensive low-density clustering occurs at lower temperatures, and ideally can be considered as icosahedral structuring (Chaplin, 2000). The expanded structure is fully tetrahedrally bonded and contains a number of clathrate cage-like structures. Such stretched 'expanded' water tends to form in small 'cavities', where strong hydrogen bonding is such as to stretch the structure, or where the clathrate cages are filled and stabilised with hydrophobic structures. This situation makes the expanded structure a better solvent for the more hydrophobic molecules. The higher density 'collapsed' structure tends to form around small highlycharged ions or where there is extensive weak hydrogen bonding. Low-density water is a good solvent for hydrophobic molecules, as they need to order the water around them. This water is already ordered in low-density water. Put simplistically, hydrophilic molecules prefer a denser aqueous environment (i.e. more water) whereas hydrophobic molecules prefer a less-dense aqueous environment (i.e. less water).

\section{Modelling of fibre effect in the colon}

How do we take advantage of these changes in water within the colon? It is clear that cancer risk must correlate with the carcinogen concentration within the mucin layer. Apart from the controlled environment within the microflora, there are three main aqueous compartments in the colon: mucus; bulk water; unfermented fibre. The mucus and dietary fibre form separate aqueous phases. Molecules partition between these environments on the basis of their hydrophile or hydrophobe properties and the properties and extents of these aqueous environments. The mucin concentration (about $2 \%(\mathrm{w} / \mathrm{w}))$ is required as a lubricant and for the protection of the mucosal surface. Briefly, the structure of the main mucus glycoprotein (MUC2; Allen et al. 1998) is a long thin charged molecule $(700 \times 10 \mathrm{~nm}$ radius $)$ that is $78 \%$ glycosylated and sulfated, particularly when found towards the distal end of the colon. As other high-density-water formers (e.g. polyacrylate, carboxymethyl cellulose) are known to be muco-adhesive, it must produce a high-density water environment due to the mass of weak hydrogen bonding and ionic effects. Bulk water forms the main aqueous environment within the colon. A fibre phase forms a third environment consisting of 'stretched' low-density water, and may be produced by partial fermentation of soluble fibre, such as the arabinoxylans, or from insoluble plant residue.

The colonic environment is modelled here using estimates for the dimensions of the aqueous compartments and assuming the amount of non-digestible and non-fermentable fibre ingested is equal to $7 \mathrm{~g} / \mathrm{d}$. Solute molecules are distributed on the basis of their preference for high-density or low-density water, dependent on the volume of the phases present (see p. 227). No direct binding of any molecules is included in the model. Estimation of the colonic compartmental volumes and surface areas (Table 2) are based on those of the 'visible man' Joseph Paul Jernigan, a slightly overweight convicted murderer. After execution, his body was frozen, sliced into $1 \mathrm{~mm}$ slices, imaged, and put on the World Wide Web (http://www.nlm.nih.gov/ research/visible/visible_gallery.html). It is further assumed that the water content on intake (1600 litres containing 1570 litres free water) is reduced (130 litres output containing 11 litres free water and $86 \mathrm{~g}$ bacteria containing about two-thirds water) and solids fermented ( $60 \mathrm{~g}$ input, $37 \mathrm{~g}$ output) within a transit time of $2 \mathrm{~d}$. As the volumes decrease through the compartments (Table 2) towards the rectum, the concentrations of solutes and the mucus thickness both increase. These variables determine the mucus volume through which any carcinogens must pass and the aqueous volumes involved in the partitioning. The results from the modelling are robust with respect to reasonable variation in the choice of these variables.

Table 2. Parameters used in the colon model, with the results of the model and the reported cancer risk for the colonic compartments*

\begin{tabular}{|c|c|c|c|c|c|}
\hline Parameters & Right & Transverse & Left & Sigmoid & Rectum \\
\hline Dimensions $($ length $\times$ diameter $; \mathrm{cm}$ ) & $24 \times 5$ & $12 \times 5+22 \times 3.3$ & $30 \times 3.3$ & $35 \times 1.9$ & $10 \times 1.9$ \\
\hline Volume (I) & 351 & 305 & 198 & 78 & 21 \\
\hline Surface $\left(\mathrm{cm}^{2}\right)$ & 377 & 416 & 311 & 209 & 60 \\
\hline Mucus depth $(\mu \mathrm{m})$ & 430 & 460 & 540 & 580 & 620 \\
\hline Cancer risk (\%) & $6 \pi$ & 8 & 6 & 26 & 42 \\
\hline Mucin hydrophobe†‡ & 0.26 & 0.43 & 0.60 & 1.83 & 1.94 \\
\hline Mucin + low fibre partition†£ & 0.26 & 0.37 & 0.46 & 1.39 & 1.47 \\
\hline Mucin + high fibre partition ${ }^{\dagger} \|$ & 0.05 & 0.05 & 0.03 & $0 \cdot 10$ & $0 \cdot 10$ \\
\hline
\end{tabular}

*The volumes and surface areas have been corrected for invaginations and turns. The transverse section was modelled in two parts and the results combined. A uniform linear flow of hydrophobic solute is assumed through all compartments with a $2 \mathrm{~d}$ transit time. For details of model, see p. 226.

†Concentration of the hydrophobic solute within the mucin layer.

$\ddagger$ Assuming a partition of 2 between the bulk water and the mucin.

\$Assuming partitions of 2 between the bulk water and the mucin and also between the water in the unfermented fibre and the bulk water.

II Assuming partitions of 10 between the bulk water and the mucin and also between the water in the unfermented fibre and the bulk water.

$\uparrow$ Cancer prevalence at the junction of the ileum and caecum has not been included. 
The potential solute carcinogens within the colon are all, at least somewhat, hydrophobic. Their hydrophobicity can be measured using the $\log \mathrm{P}$ parameter (Leo, 1993), determined from their partition coefficients between water and noctanol, where any $\log \mathrm{P}$ above zero indicates a net hydrophobic character with higher $\log \mathrm{P}$ indicating greater hydrophobicity. Typical potential or actual carcinogens are 3,4-benzpyrene (a polycyclic aromatic hydrocarbon from smoked foods; $\log \mathrm{P} 4.0$ ), Trp-P-1 (a heterocyclic aromatic hydrocarbon derived from tryptophan in cooked red meat and other high-quality protein; $\log \mathrm{P} 1 \cdot 8$ ), fecapentaene-12 (produced by anaerobic organisms from bile acids; $\log \mathrm{P}$ $0 \cdot 7$ ), N-nitrosodimethylamine (from protein and nitrates; $\log \mathrm{P} 1.4$ ), and lithocholic acid (a natural co-carcinogen; $\log \mathrm{P} 3 \cdot 5)$. Such molecules will partition between the three aqueous compartments in the colon, with partition coefficients that depend on their hydrophobicity: (1) mucus containing higher-density water (HDW), having volume $\mathrm{V}_{\mathrm{HDW}}$ containing solute $\mathrm{X}$ with concentration $\mathrm{X}_{\mathrm{HDW}}$; (2) fibre residue containing lower-density water (LDW), having volume $\mathrm{V}_{\mathrm{LDW}}$ containing solute $\mathrm{X}$ with concentration $\mathrm{X}_{\mathrm{LDW}}$; (3) the remaining water containing normal-density water (NDW), having volume $\mathrm{V}_{\mathrm{NDW}}$ containing solute $\mathrm{X}$ with concentration $\mathrm{X}_{\mathrm{NDW}} . \mathrm{P}_{\text {in }}$ and $\mathrm{P}_{\text {out }}$ are partition coefficients.

$$
\begin{gathered}
P_{\text {in }}=\frac{X_{H D W}}{X_{N D W}} \quad P_{\text {out }}=\frac{X_{L D W}}{X_{N D W}} \\
X_{H D W}=\frac{X}{V_{H D W}+V_{N D W} \times \frac{1}{P_{\text {in }}}+V_{\text {LDW }} \times \frac{P_{\text {out }}}{P_{\text {in }}}} \\
X_{\text {NDW }}=\frac{X}{\mathrm{~V}_{\text {HDW }} \times P_{\text {in }}+V_{N D W}+V_{\text {LDW }} \times P_{\text {out }}} \\
X_{\text {LDW }}=\frac{X}{V_{\text {HDW }} \times \frac{P_{\text {in }}}{P_{\text {out }}}+V_{\text {NDW }} \times \frac{1}{P_{\text {out }}}+V_{\text {LDW }}}
\end{gathered}
$$

Thus, all these molecules should have a concentration ratio between the high-density water of the mucus and the low-density water associated with the fibre of between about 5 and $>100$ in favour of the fibre environment.

The results of this model (Table 2) indicate that the partitioning of hydrophobic molecules within the colon towards the unfermented dietary fibre phase will operate to reduce their concentration within the mucus layer. This reduction will be particularly noticeable towards the end of the colon where the prevalence of carcinomas is greatest.

\section{Conclusion}

Entrapped water is very important in removing hydrophobic molecules from the colon, particularly if it has low-density character. Particles, or low-density water-forming gels, can entrap water best. Partition of hydrophobic carcinogens or potential carcinogens away from the mucosal surface can be achieved by the presence of unfermented dietary fibre, which may explain at least some of its beneficial properties. One interesting question is whether exercise encourages the partition process?

\section{References}

Allen A, Hutton DA \& Pearson JP (1998) The Muc2 gene product: a human intestinal mucin. International Journal of Biochemistry and Cell Biology 30, 797-801.

Ang JF (1991) Water-retention capacity and viscosity effect of powdered cellulose. Journal of Food Science 56, 1682-1684.

Auffret A, Ralet MC, Guillon F, Barry JL \& Thibault JF (1994) Effect of grinding and experimental conditions on the measurement of hydration properties of dietary-fibers. Food Science and Technology - Lebensmittel-Wissenschaft und Technologie 27, 166-172.

Bellissent-Funel M-C (2001) Structure of confined water. Journal of Physics: Condensed Matter 13, 9165-9177.

Blackwood AD, Salter J, Dettmar PW \& Chaplin MF (2000) Dietary fibre, physicochemical properties and their relationship to health. Journal of the Royal Society for the Promotion of Health 120, 242-247.

Bodor N, Gabanyi Z \& Wong CJ (1989) A new method for the estimation of partition-coefficient. Journal of the American Chemical Society 111, 3783-3786.

Chaplin MF (2000) A proposal for the structuring of water. Biophysical Chemistry 83, 211-221.

Chaplin MF (2001) Water; its importance to life. Biochemical and Molecular Biology Education 29, 54-59 (developed further at http://www.sbu.ac.uk/water/).

Chen JM, Kung CE, Feairheller SE \& Brown EM (1991) An energetic evaluation of a Smith collagen microfibril model. Journal of Protein Chemistry 10, 535-552.

Devlin JP (2000) Preferential deuterium bonding at the ice surface: A probe of surface water molecule mobility. Journal of Chemical Physics 112, 5527-5529.

Durchschlag H \& Zipper P (2001) Comparative investigations of biopolymer hydration by physicochemical and modelling techniques. Biophysical Chemistry 93, 141-157.

Guillon F, Renard CMGC, Hospers J, Thibault JF \& Barry JL (1995) Characterization of residual fibers from fermentation of pea and apple fibers by human fecal bacteria. Journal of the Science of Food and Agriculture 68, 521-529.

Hayashi Y, Shinyashiki N \& Yagihara S (2002) Dynamical structure of water around biopolymers investigated by microwave dielectric measurements using time domain reflectometry method. Journal of Non-Crystalline Solids 305, 328-332.

Hill MJ (1999) Mechanisms of diet and colon carcinogenesis. European Journal of Cancer Prevention 8, S95-S98.

Janaswamy S \& Chandrasekaran R (2001) Three-dimensional structure of the sodium salt of iota-carrageenan. Carbohydrate Research 335, 181-194.

Leo AJ (1993) Calculating Log P(oct) from structures. Chemical Reviews 93, 1281-1306.

Nieduszynski IA \& Marchessault RH (1972) Structure of $\beta$-D(1-4)xylan hydrate. Biopolymers 11, 1335-1344.

Thebaudin JY, Lefebvre AC, Harrington M \& Bourgeois CM (1997) Dietary fibres: Nutritional and technological interest. Trends in Food Science and Technology 8, 41-48.

Wiggins PM (1995) High and low-density water in gels. Progress in Polymer Science 20, 1121-1163. 